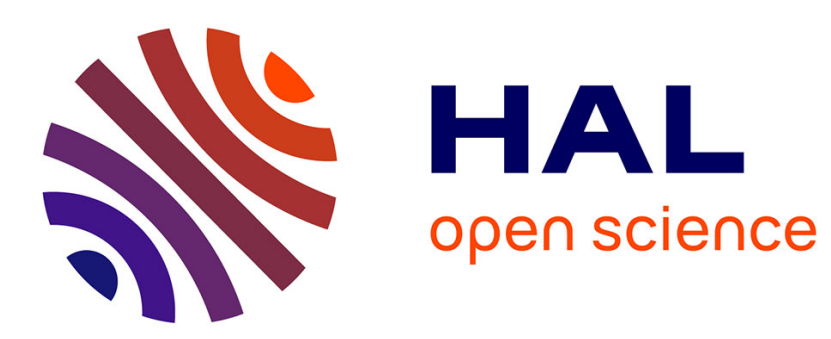

\title{
Succession des banlieues et forme de la croissance urbaine dans l'ouest nantais \\ Daniel Pinson
}

\section{To cite this version:}

Daniel Pinson. Succession des banlieues et forme de la croissance urbaine dans l'ouest nantais. Villes en parallèle, 1990, Peuplements en banlieue, 15 (n¹5-16), p. 32-57. 10.3406/vilpa.1990.1074 . halshs01532761

\section{HAL Id: halshs-01532761 https://shs.hal.science/halshs-01532761}

Submitted on 19 Oct 2018

HAL is a multi-disciplinary open access archive for the deposit and dissemination of scientific research documents, whether they are published or not. The documents may come from teaching and research institutions in France or abroad, or from public or private research centers.
L'archive ouverte pluridisciplinaire HAL, est destinée au dépôt et à la diffusion de documents scientifiques de niveau recherche, publiés ou non, émanant des établissements d'enseignement et de recherche français ou étrangers, des laboratoires publics ou privés. 


\title{
Succession des banlieues et forme de la croissance urbaine dans l'Ouest nantais
}

Daniel Pinson

\section{Résumé}

Observation, sur la longue durée, de la poussée nantaise vers l'Ouest, permettant d'analyser, dans leur double dimension démographique et urbanistique, les rapports entre ville-centre et banlieue en fonction de l'évolution des différents facteurs de croissance et des politiques urbaines. La méthode envisage certains rapprochements dans le mode de peuplement des deux communes concernées, permettant une réflexion sur la façon dont se combinent, dans le processus d'extension urbaine, les facteurs économiques, démographiques, politiques et urbanistiques.

\begin{abstract}
This observation, of long duration, of the population movements towards the West of Nantes allows one to analyse, in their double demographic and urban dimension, the relations between city-centre and suburbs in regard to the evolution of different development factors and town planning policies. The method sees certain similitudes in the manner of populating the two communes of interest permetting thought about the way economic, demographic, political and urban factors combine in the process of urban expansion.
\end{abstract}

\section{Citer ce document / Cite this document :}

Pinson Daniel. Succession des banlieues et forme de la croissance urbaine dans l'Ouest nantais . In: Villes en parallèle, $\mathrm{n}^{\circ} 15-$ 16, juin 1990. Peuplements en banlieue. pp. 32-57;

doi : https://doi.org/10.3406/vilpa.1990.1074

https://www.persee.fr/doc/vilpa_0242-2794_1990_num_15_1_1074

Fichier pdf généré le 24/05/2018 


\section{Résumé}

Observation, sur la longue durée, de la poussée nantaise vers l'Ouest, permettant d'analyser, dans leur double dimension démographique et urbanistique, les rapports entre ville-centre et banlieue en fonction de l'évolution des différents facteurs de croissance et des politiques urbaines. La méthode envisage certains rapprochements dans le mode de peuplement des deux communes concernées, permettant une réflexion sur la façon dont se combinent, dans le processus d'extension urbaine, les facteurs économiques, démographiques, politiques et urbanistiques.

\section{Abstract}

Suburbs and Urban Expansion in the West of Nantes (Chantenay-XIX ${ }^{\text {th }}$, St Herblain-XX ${ }^{\text {th }}$ )

This observation, of long duration, of the population movements towards the West of Nantes allows one to analyse, in their double demographic and urban dimension, the relations between city-centre and suburbs in regard to the evolution of different development factors and town planning policies. The method sees certain similitudes in the manner of populating the two communes of interest permetting thought about the way economic, demographic, political and urban factors combine in the process of urban expansion. 


\section{SUCCESSION DES BANLIEUES \\ ET FORME DE LA CROISSANCE URBAINE DANS L'OUEST NANTAIS}

Daniel PINSON*

La croissance de l'agglomération nantaise a été depuis longtemps orientée vers l'Ouest, remontant de siècle en siècle, et de décennic en décennie, vers l'embouchure de la Loire. Dès le XVII siècle, la formation du Quai de la Fosse en constitue l'illustration, étendant les parties urbanisées de la ville de Nantes jusqu'aux portes de Chantenay. Cette commune, antichambre industrielle de la grande ville, n'a guère plus d'un siècle d'existence autonome puisqu'elle fut annexée à Nantes en 1908. Le XXc siècle a encore confirmé cette poussée vers l'Ouest, tempérée seulement dans la deuxième moitié du siècle par les mesures volontaristes de l'aménagement du territoire (SDAAM et SDAU) et un retournement relatif de l'activité économique nantaise vers l'intérieur du territoire national (années 60).

Observée sur la longue durée, cette croissance occidentale se révèle intéressante : deux communes de banlieue seront successivement concernées par la poussée d'urbanisation nantaise : Chantenay au XIXe siècle, et St-Herblain au XX' $X^{\mathrm{c}}$. L'évolution des différents facteurs de la croissance, comme celle des politiques urbaines introduisent nécessairement de profondes distinctions dans les rythmes de peuplement et la nature des rapports entre ville-centre et banlieue. Ce sont ces rapports dans leur double dimension démographique et urbanistique que nous nous proposons d'analyser ici : nous essaierons

\footnotetext{
* Architecte et sociologue, C.N.R.S., Laboratoire d'Études et de Recherches Sociologiques sur la Classe Ouvrière, Nantes
} 
de cerner l'arrivéc des populations, leur établissement dans l'espace urbain et l'effet de l'économie et de la gestion urbaine dans les modes d'établissement. Déroulant l'observation sur l'axe du temps, la méthode utilisée n'a aucune visée comparative, au plus envisagera-t-elle certains rapprochements dans les modes de peuplement des deux communes, permettant d'amorcer un certain nombre de réflexions sur la façon dont se combinent, dans le processus d'extension urbaine, les différents facteurs économiques, démographiques, politiques et urbanistiques.

Les éléments produits ici s'appuicront sur deux recherches, la première portant sur la totalité de la commune de Chantenay, essentiellement appréhendée dans sa période d'indépendance ${ }^{1}$, l'autre sur la ZUP de Nantes-St-Herblain, vaste ensemble résidentiel de 7500 logements étudié dans le contexte du développement urbain des années $60^{2}$.

Chantenay, sur l'axe de développement occidental de l'agglomération nantaise, est spatialement une banlieue de la première couronne et historiquement une banlicue de la première ère du développement industricl de Nantes, au XIX ${ }^{\mathrm{e}}$ siècle. St-Herblain appartient à la deuxième couronne et correspond au fort développement économique et urbain de la deuxième moitié du XXe siècle, celui des "trente glorieuses".

Quelques données chiffrées permettent de mieux saisir ces évolutions. De 1806 à 1906, les populations de Nantes et de Chantenay augmentent considérablement : Nantes double sa population (de 65000 à 133000 ), tandis que Chantenay la décuple (1 800 à 21380 ). Saint-Herblain est une commune rurale dont la population reste stable (2 300 habitants en 1806, 2500 habitants en 1906).

Nantes doublera encore sa population au XXe siècle, passant à 260000 habitants en 1968, mais l'essenticl de cette augmentation est réalisé après la seconde guerre mondiale (1946 : 188123 habitants). 1968 est en même temps le point culminant de la croissance démographique nantaise, son dépeuplement atteignait 20000 habitants $(240000)$ en 1982.

Simultanément, Saint-Herblain voyait sa population passer de 2 à 500 habitants au début du siècle à près de 42000 en 1982, l'essentiel de cette augmentation se réalisant cntre 1954 et 1968 (de 7636 à 18760 habitants) et de 1968 à 1975 (de 18760 à 39867 habitants). 
Ces rythmes sont, pour les deux communes de Chantenay et de S xint-Herblain, très spectaculaires, mais à la vérité, celui qui touche Saint-Herblain, l'est encore plus et s'explique largement par l'érection de la ZUP de Nantes-St-Herblain, créant sur cette dernière commune un apport de 10000 habitants en l'espace de deux recensements (1968-1975). On a là un rythme de croissance urbaine que le XIXe siècle n'a pas connu dans la région nantaise. Deux décennies du XIX ${ }^{\mathrm{e}}$ siècle marquent pourtant à Chantenay des bonds qualitatifs substanticls en matière démographique : 1851-1861 et 1891-1901, où la population passe respectivement de 5000 à 9000 habitants et de 13800 à 19640 . Ces poussées démographiques recouvrent, avec des écarts spécifiques régionaux, des phases de croissances similaires constatées au niveau national ${ }^{3}$.

Ces données étant ainsi présentées, revenons à présent à une appréhension plus continue du développement de l'agglomération, en suivant le temps de l'histoire urbaine. Notre perspective est maintenant de cerner la nature et la localisation des installations résidenticlles et d'en voir le rapport avec l'économique et le politique.

\section{Q CHANTENAY}

\section{Les usines sortent de Nantes : Chantenay, banlieue industrielle}

Le développement de Chantenay, dans les premières décennies du XIX ${ }^{\mathrm{e}}$ siècle, résulte principalement de l'implantation, hors du territoire nantais, d'industries exigeant des sites appropriés à leur activité. Ce seront, d'une part, les chantiers navals qui ne trouvent plus suffisamment de place à Nantes (les terrains au bord du fleuve sont comptés !), et d'autre part, les conserveries dont les exhalaisons incitent les proprićtaires à repousser leurs établissements toujours plus loin des zones habitécs. Ce sont véritablement ces deux industries qui crécront, à la limite de Nantes, deux gros faubourgs (La Grenouillère dans le Bas Chantenay et la Ville en Bois dans le Haut Chantenay (fiefs respectifs de la Construction navale et de la Conserverie).

Avant l'arrivée des usines et chantiers, on peut en effet considérer que Chantenay reste encore un bourg rural, parsemé de hameaux dont certains subissent déjà une certaine attraction nantaise. C'est ce dont témoigne l'un des premiers maires de Chantenay disant, dans un courrier au Préfet daté de 1812, que la plupart des habitants de sa commune sont 
des "ouvriers et des portefaix", alors qu'à St-Herblain les gens sont plus à l'aise, "étant tous de bons fermiers et laboureurs". Mais c'est sans doute véritablement les implantations industrielles qui amorcent la mutation de Chantenay de commune rurale en banlieue urbaine.

Les ateliers et chantiers ne dépassent guère la cinquantaine d'ouvriers et se présentent souvent, lorsqu'il s'agit des conserveries, comme de grandes maisons à rez-dechaussée commercial, qui développent en fond de parcelle des halls et appentis abritant les opérations de fabrication.

\section{Les ouvriers s'agglomèrent autour des usines}

La première installation des usines ne survient jamais dans un paysage complètement vierge. S'ajoutent très rapidement des immeubles d'un ou deux étages avec combles. abritant des commerces et des locaux d'habitation. Leur multiplication est favorisée par la présence de l'usine et de son activité périphérique (embauche/débauche) mais aussi par le fait qu'on se trouve là en deçà des limites de la ville de Nantes et que les taxes d'octroi sur les boissons, l'alimentation et même les matériaux de construction sont nettement moins élevées à Chantenay ${ }^{4}$.

\section{Les vagues de peuplement : $1850-1860,1890-1900$}

La décennie 1850-1860 marque, pour Chantenay, l'un des plus forts moments de sa croissance démographique ; sa population double de 1851 a 1861 (4966 habitants en 1851, 9066 en 1861). Cet espace intercensitaire se situe dans la première moitié du Sccond Empire, marquée nationalement par un fort développement économique. Chantenay bénéficie amplement de cet essor avec l'ouverture de la voie ferrée, prolongeant le chemin de fer (arrivant de Paris à Nantes) vers le chapelet des ports de la côte sud de la Bretagne : Quiberon, Port-Louis, Étel, Lorient, Vannes. Une chaîne industrielle se développe autour des activités de la mer et de l'agriculture. Les conserveries appellent les fabriques de boîtes de conserves, la raffinerie de sucre nécessite le "noir animal" qui trouve de nouveaux usages comme engrais pour l'agriculture. Les usines se multiplient en même temps qu'elles augmentent en taille.

La dernière décennie du XIXc siècle voit également, à la faveur d'un nouveau développement économique, un autre bond dans l'accroissement de la population chantenay- 
sienne. Entre 1891 et 1901 , elle augmente de $42 \%$ portant le nombre d'habitants de Chantenay à près de 20000 (19 640). Ce pourcentage contraste avec les précédents qui, entre deux dénombrements décennaux, ne dépassaient jamais $20 \%$. La brutalité de cette augmentation rend critique la situation du logement ouvrier dans la commune, à la fois sur le plan du besoin et de l'hygiène, nous allons y revenir.

\section{L'origine des apports de population}

Plus que les dénombrements qui mentionnent rarement le lieu de naissance, les listes électorales donnent une bonne idée de l'origine des nouveaux habitants. On s'en doute, l'accroissement de la population n'est pas issu principalement de l'excédent naturel de la population communale, mais des apports migratoires. C'est surtout le département de Loire-Inféricure qui semble le plus gros fournisseur. Vers 1901, 60\% des électeurs ont une naissance dans ce département, tandis que les Bretons et les Vendéens d'origine sont dans la proportion de $9 \%$ et $5 \%$.

\section{Les lieux de l'établissement ouvrier : de l'intrication à la séparation}

Lors de la première vague de l'industrialisation, on constate une forte intrication de l'usine et de l'habitat. L'établissement industriel n'est au départ qu'un atelier en rez-dechaussée d'immeuble qui s'étend progressivement, par adjonction successive de bâtiments, vers le fond de la parcelle d'implantation. Les décrets sur l'insalubrité (1810), la réglementation sur l'installation des machines à vapeur (1840), qui ont souvent été à l'origine de l'établissement des usines sur le site peu urbanisé de Chantenay, empêchent rarement l'érection d'immeubles d'habitation à proximité des usines ${ }^{5}$.

A la fin du XIXe, la densification progressive du tissu d'habitat et d'industric, nourri de l'extension des usines et de l'édification toujours plus serrée des maisons le long des voies existantes, finira par provoquer le déménagement de beaucoup d'usines. Ce phénomène est particulièrement sensible à la Ville en Bois, dans le Haut Chantenay, au point qu'apparaîtra avec le second essor industriel de la fin du siècle une spécialisation dans l'usage du territoire communal : les usines tendront à former une véritable zone industrielle sans le dire dans le Bas Chantenay (en prolongement du quartier de la Grenouillère), tandis que les quartiers résidentiels auront tendance à se constituer "sur les hauteurs de Chantenay, dans des conditions favorahles d'aćration" comme le précisera l'industricl Louis Amicux ${ }^{6}$. 


\section{Croissance urbaine dans l'Ouest nantais}

\section{Le mode d'urbanisation}

Dans les premiers temps et cela jusqu'en 1880 , les voies existantes, les routes qui conduisent de Nantes à St-Herblain, Coueron, Saint-Nazaire et Pontchâteau vers l'estuaire de la Loire, suffisent à l'urbanisation. L'examen des plans, malgré son manque d'homogénéité permet d'en faire le constat : les voies principales se bordent de constructions en ordre de plus en plus serré. Les techniques essentielles d'aménagement consistent, à cette époque, en alignements et nivellements : les premiers permettent l'êlargissement modéré des voies pour répondre à l'accroissement du trafic, tandis que les seconds remédient aux difficultés d'écoulement des eaux rejetées par les habitations, plus abondantes avec l'accroissement de la densité des constructions. En cette première période de l'urbanisation la construction de l'habitation est essentiellement du ressort de l'initiative privée, consistant en de très modestes immeubles de rapport.

Dans les années 1860, la création de la paroisse de St-Clair se concrétise par la construction de l'église, mais aussi par l'ouverture d'une rue axée sur sa façade et reliéc à la rue de la Ville-en-Bois, amorce de la route qui conduit à Coueron. Ce modeste percement inaugure, en même temps qu'il permet d'autres constructions en bordure de voie, un mode d'urbanisation nouveau pour Chantenay.

A partir de 1880, un boulcvard de ccinturc scra créć par la Ville de Nantes, et accepté par Chantenay. Il ouvre de nouvelles possibilités pour la construction des "maisons" (sont ainsi désignés aussi bien les petits immeubles que les maisons urbaines) alignées sur la voie. Mais il est aussi la manifestation des visées de la grande ville sur sa banlieue et constitue un aménagement plus à sa dimension qu'à celle de Chantenay. Il servira d'ailleurs d'argument plus tard pour l'annexion de Chantenay (1908).

\section{La production du logement}

Pourtant, le percement de cette grande artère, l'aménagement des places, qui rectifient les pattes d'oies à l'intersection du nouveau boulevard avec les autres voies, ne suffisent plus, à la fin du siècle, à faire face à la brutale augmentation du nombre de chantenaysiens. Ce n'est pas tant la production des voies nouvelles qui fait problème que le manque de logements et l'excessive densification de certains quartiers à proximité des usines les plus anciennes, souvent les plus nuisantes.

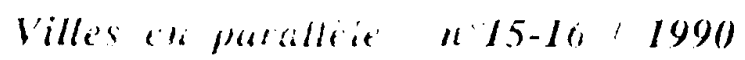


Un rapport est établi par le docteur REDUREAU à la demande de la Commission HBM constituée dès 1896 à l'initiative de plusicurs industriels de Chantenay. En même temps qu'il traduit un nouvel état d'esprit, inspiré par la loi SIEGFRIED, il témoigne de l'acuité du problème à Chantenay. Dans ce rapport sont à la fois soulignés l'insalubrité de nombreux logements et le manque de zones ouvertes à la consuruction de logements populaires.

L'accès de l'ingénieur radical-socialiste GRIVEAUD à la tête de la municipalité en 1901 conduira à une prise en main plus active de ces problèmes : ainsi, à la faveur de l'érection de la nouvelle Mairie (1904), sera également créé un vaste lotissement municipal constitué de six voies nouvelles axées sur la place de la Nation. Ce projet, réalisé, en même temps qu'il donnait à Chantenay une mairie digne de son nouvel état de ville de plus de 20000 habitants, assurait la continuité de son urbanisation jusque là écarteléc entre les concentrations du Haut Chantenay (La Ville en Bois) et du Bas Chantenay (La Grenouillère). En même temps apparaissent quelques rares cités HBM ; la première, créćc au Bois-Hardy en 1905, offre une quarantaine de maisons, mais la forme modale du logement restera jusqu'à la gucrre, à Chantenay, le petit immeuble de rapport ou la petite maisonnette individuclle.

\section{L'équipement urbain}

A vrai dire, la production du logement ne pouvait plus constituer la seule préoccupation de la ville importante qu'était désormais devenue Chantenay. Un problème que l'on appellerait aujourd'hui de "génie urbain" se posait désormais aux responsables de la conduite des affaires municipales.

La densité urbaine acquise exigeait que l'on aille au-delà des simples alignements, nivellements el percements : il fallait désormais équiper la ville : écouler les caux résiduclles, assainir les voies et ies éclairer, remplacer les puits par le service d'eau potable et installer l'éclairage public. Toutes ces techniques nouvelles, Nantes commençait à les maîtriser. A Chantenay, la nécessité de leur application ne devint évidente qu'avec le fort accroissement démographique de la fin du siècle et l'état désastreux de la situation sanitaire qu'il révéla dans certains quartiers.

Une convention avait été signée en 1896 qui permit à la ville de Nantes d'édifier entre 1902 et 1904, sur le reiicf le plus élevé de l'aggloméraiion, situé à Chantenay, un ré- 
servoir d'eau potable. En contrepartie, Nantes s'engageait à alimenter les parties les plus habitées de Chantenay, celles qui, précisément, se trouvaient à la Ville en Bois comprises entre les limites communales de Nantes et de Chantenay et le boulevard de ceinture. Avec celui-ci, entretenu et éclairé aux frais de la grande ville, la mise en place de ce réseau fournit un argument supplémentaire à l'annexion de Chantenay.

\section{Une urbanisation hétéroclite}

A la fin du $\mathrm{XIX}^{\mathrm{e}}$ siècle, le territoire de Chantenay présentait une répartition très déséquilibrée : les quartiers les plus urbanisés étaient situés a la lisière nantaise. Cette densification était également encouragée par la desserte du Tramway à air comprimé, facilitant l'accès au centre de la ville et permettant une mobilité autre que pédestre. Celle-ci restait pourtant le lot de la plupart des habitants de Chantenay, ce qui, sans doute, dissuadait des choix résidentiels trop éloignés du lieu de travail et compromettait une urbanisation plus étalée de la commune.

Les petits immeubles de rapport dominaient dans les quartiers les plus urbanisés, réalisant un front continu le long des voies, tandis que des maisons ouvrières isolées commençaient à se construire dans les quelques lotissements créés, comme à la Villanauva et le long du chemin des Pavillons, dans des parties plus centrales du territoire de la commune. Souvent, même dans les zones les plus urbanisées, le maillage peu serré des voies bordées d'habitations laissait de vastes terrains enclavés qui recevaient quelques constructions isolées et plus souvent des jardins ouvriers comme aux Courtils et à la Fournillère.

Ainsi, Chantenay présentait-il, au début de ce siècle, un paysage très varié et changeant, à quelques centaines de mètres et d'un endroit à l'autre, de rues très urbaines, de lotissements bien tracés, de bouts de campagnes isolées au milicu des maisons, et d'usines encore mêlées au tissu urbain comme à la Ville en Bois (Haut Chantenay) ou rassemblées dans une vaste zone industrielle (le Bas Chantenay). Un paysage de transitions finalement assez propice à la vic nouvelle d'une population dont l'origine rurale n'était pas encore aussi lointaine dans la plupart des cas.

La mise en place de ce paysage résulte de la combinaison de multiples initiatives successives, d'abord celle des industriels, puis celle des commerçants, des propriétaires, des spéculateurs, et dans un dernier temps, celles de sociétés HBM et de la municipalité. 
Elles s'adaptent d'abord aux potentialités existantes du site : voies et villages existants, engendrant la densification des derniers et la formation de "doigts de gants" le long des premières. Ce n'est que dans un second temps, à la fin du XIXe siècle, qu'apparaissent des initiatives plus globales impliquant, pour la création d'ensembles de logements plus importants, l'acquisition de parcelles plus vastes ou plus nombreuses, le tracé et la création de voies rationnellement organisées pour le lotissement, selon un maillage à géométrie fortement organisée.

\section{Stagnation nantaise de l'entre-deux-guerres}

Après les rattachements de Chantenay et Doulon, la croissance de Nantes sera, dans l'entre-guerres, relativement modeste, plus faible que la moyenne nationale.

\section{Le bond de l'après-guerre}

C'est après la seconde guerre mondiale que la Ville de Nantes va voir un fort développement de sa population. Entre 1946 et 1962, elle passe de 190000 habitants à 240 000. La ville participe, même si cela est dans une moindre mesure, au fort redéploiement économique de l'après-guerre et l'on assiste à une importante immigration des campagnes environnantes. Alors qu'en 1931, la population urbaine du département de Loire-Atlantique n'atteignait que $42 \%$ de la population totale, contre $51 \%$ pour l'ensemble de la France, elle rejoint en 1962 la moyenne nationale avec $63 \%$. En plus du croît naturel, cette augmentation bénéficie d'un solde migratoire positif important à la différence des départements voisins.

\section{La poursuite des lotissements}

Un décalage se creuse peu à peu entre le besoin de logements nouveaux ếl'offre qu'apportc la ville : la production des lotissements et des pavillons se pourstit à un rythme comparable à celui de l'entre-deux-guerres, malgré l'entrée en lice de quelques nouveaux promoteurs sociaux. Ainsi, un paysage pavillonnaire tend désormais à remplir les espaces libres de Chantenay et à gagner déjà la commune de St-Herblain, le long de la route qui mène à Vannes, en lisière des villes de Nantes et d'Orvault. En 1962, StHerblain doublait bientôt sa population de 1946 (12 000 habitants contre 5500$)^{7}$.

L'aggravation des conditions de logement dans la ville-centre et l'incapacité relative des lotissements à résoudre le besoin de logements né de la poussée démographique de l'après-guerre, va alors favoriser le recours à la politique des grands ensembles. 


\section{DAINT-HERBLAIN}

\section{La création de la ZUP de Nantes-Saint-Herblain}

La ZUP de Nantes-St-Herblain (7 500) logements) est créée par arrêté ministéricl du 7 octobre 1959 (la loi sur les ZUP date du 31 XII 1957), l'opération a été conçuc et préparée par la Direction Départementale de l'Équipement, saus que les communes concernées aient véritablement été consultées, nous allons y revenir.

La situation du loggement dans l'agglomération nantaise, a fait l'objet d'une étude qui fonde les arguments essentiels de la programmation de la ZUP. Il y est ainsi précisé que 20000 ménages vivent à Nantes en condition de surpeuplement et que par ailleurs 400() familles habitent encore des taudis ou des baraquements et que 1000 autres encore sont logées dans des immeubles vétustes. "Pour résorber la crise du logement sur 10 ans (perspective 1970), dit encore une note d'explication à la création de la ZUP\$, il est nécessaire de créer 30000 logements au rythme de 3000 par an."

\section{La croisade anti-pavillonnaire}

Désormais la solution à cette production en grand nombre de logements impose, selon l'administration de l'État, le recours à l'immeuble collectif industrialisć. Les thèses défendues par les partisans de la Charte d'Athènes triomphent et l'expérience acquise dans la réalisation des grands ensembles encourage à aller encore plus loin dans le gigantisme. Parmi les justifications avancées en faveur de la ZUP, un argument significatif de cette idéologic antipavillonnaire est mis en avant avec force : "mettre un terme au développement anarchique de la partie Ouest de l'agglomération nantaise". Cette assertion concerne bien entendu toute la zone qui englobe l'ancienne commune de Chantenay et les abords de St-Herblain, et vise les développements pavillonnaires qui s'y sont réalisés à la fin du XIXe siècle et entre les deux conflits mondiaux.

\section{L'emplacement de la ZUP}

Asse\% curieusement, dans la documentation consultée sur la création de la ZUP (Archives de la Société d'Équipement et délibérations des conscils municipaux) ${ }^{9}$, il est fait peu de cas du rapport entre la création de la ZLjP et la localisation des zones d'emploi. Les justifications de son emplacement sont essentiellement rapportées à des considéra- 
tions urbanistiques générales, (le "développement anarchique de l'ouest de l'agglomération"), topographiques et techniques ("exposition remarquable des terrains ct bons sols", "facilité d'équipement") et financière ("coût d'acquisition bas").

En réalité, les responsables de l'aménagement, et d'abord ceux qui gravitent dans les administrations de l'État, semblent bien avoir la conviction que scules les ZUP constituent les instruments adéquats à la remise en ordre de la ville. En gelant des réserves foncières importantes, on pense arrêter la spéculation et le gaspillage des lotissements; en concentrant les opérations, on estime se donner les moyens de répondre rapidement à la forte demande de logements sociaux.

Car la croisade contre les lotissements et les pavillons se double aussi d'un combat en faveur de l'unité de la ville, contre l'esprit de clocher qui dresse les banlieues contre la ville-centre, empêchant l'adoption d'aménagements profitables à l'ensemble de l'agglomération.

C'est peut-être sous cet angle qu'il faut analyser la décision d'un tracé de la ZUP s'inscrivant sur les deux communes de Nantes et de St-Herblain. D'ailleurs le cas n'est pas isolé : il concerne plusieurs grands ensembles de la région parisienne et selon E. PRETECEILLE visait à favoriser la fusion des Communes ou à crécr unc structure administrative les chapeautant ${ }^{10}$. Ce projet existait également dans l'agglomération nantaise et plusicurs tentatives préfectorales allant dans ce sens (1966 ct 1972) furent finalement repoussées par les maires de la plupart des villes de banlieuc.

L'idée d'un continuum urbain, s'attachant à la compacité du tissu urbain depuis le cocur de la ville-centre, était l'obsession des aménageurs. Dans le cadre d'une telle conception, l'existence des limites communales, des territoires, icur histoire et leur identité étaient perçues comme des données anachroniques que le développement de l'agglomération rendait caduques. Dans cette perspective, l'agglomération se confond d'ailleurs sur la ville-centre : celle-ci est le moteur du dévcloppement urbain, en conséquence la cohérence de son territoire a une priorité légitime sur celui des communes limitrophes.

A cet endroit, on peut, sans doute, faire un lien entre la volonté expansionniste nantaise au XIXe siècle, ses préparatifs (la création du boulevard de ceinture, véritable corde au cou de Chantenay puisque tracé sur son territoire, létablissement du réservoir 
d'eau potable pour l'alimeptation de la ville, également édifié sur Chantenay), sa conclusion (l'annexion de 1908), et la création par l'État des années 60 d'une structure d'agglomération dominant les institutions municipales existantes. D'une certaine manière, la ZUP de Nantes-St-Herblain jouait dans cette perspective le rôle qu'avait joué le boulevard de ceinture dans l'extension territoriale nantaise. Mais, à la différence, ce n'est plus la grande ville qui est maître du jeu, mais véritablement l'État.

\section{Préparation technocratique des isolats dans la ZUP}

C'est en effet l'État qui décréte la ZUP, mais en définit également la situation, le contenu, et la réalisation. C'est ainsi que l'adjoint au maire de Nantes, Président de l'Office HLM, s'entend dire qu'aucune de ses opérations ne bénéficiera des subventions et prêts de l'État en dehors de la ZUP. Bientôt les pouvoirs des municipalités seront délégués à la société d'économie mixte créée à l'initiative de l'État (la SÉLA) pour la conception de la ZUP (puis sa réalisation) et d'autres grandes opérations d'aménagement à venir. La Caisse des Dépôts y détient un maximum de pouvoirs et saura y exercer son autorité, imposant l'Architecte en Chef, le programme et le modèle architectural et urbanistique de la ZUP : elle tient les cordons de la Bourse et dispose des prétentions de compétence de ses techniciens.

C'est donc toute une conception de la croissance urbaine qui s'impose à la Ville de Nantes, à sa banlieue, St-Herblain, et va de cette manière encadrer le mode de répartition des populations mal logées ou en demande de logement.

Malgré les déclarations d'intention, affirmées par la Préfecture et la SÉLA, de faire obstacle à la ségrégation sociospatiale en favorisant des opérations autres qu'HLM, le mode de répartition, les rigidités administratives ${ }^{11}$ et l'ampleur des programmes vont conduire à la formation d'isolats dont le plus visible est celui constitué sur la partie Est de la ZUP par l'ensemble de l'Office Public HLM de la Ville de Nantes. En effet, l'artifice des 4 tranches d'environ 500 logements ne peut masquer le regroupement effectif du parc de l'Office Municipal, tandis qu'à St-Herblain l'alternance des tranches de logements sociaux des sociétés coopératives ou anonymes HLM et des ensembles privés en accession atténue la dimension des plus grandes opérations (500 logements). 


\section{Le peuplement d'origine de la ZUP}

Ainsi, la livraison des premiers appartements, dès 1965, permettait de satisfaire le relogement des populations qu'avaient ciblées les autorités municipales et préfectorales, en particulier celles qui vivaient dans les baraquements d'urgence montés après la guerre au Nord et à l'Est de Nantes (la Petite Sensive et le Grand Blottereau) et les jeunes ménages qui vivaient dans les immeubles vétustes du centre-ville ou cohabitaient encore avec leurs parents. A ces deux types de population correspondaient les nouvelles catégories de logements définis par l'administration les PSR (programmes sociaux de relogement) et les HLM ordinaires qui allaient encore créer dans la concentration des HLM de la Ville de Nantes des isolats supplémentaires.

Les années 70 donnent pour la ZUP une structure de population en énorme contraste avec les moyennes nationales et urbaines. Jeunes ménages et familles nombreuses y dominent largement, conduisant dans les années 75 à une forte proportion d'adolescents et conjointement à une absence notoire de personnes âgées.

Dans le recensement de 1975 , les tranches d'âge de 0 à 19 ans compteront pour $47 \%$ sur la partie nantaise de la ZUP et pour $49 \%$ sur la partie herblinoise, ce qui est à comparer avec la moyenne de $31 \%$, valant pour la France entière. A l'autre extrémité, les plus de 65 ans ne totalisent pas plus de $3 \%$ de la population alors qu'ils comptent pour $14 \%$ dans la France entière.

Cette structure est indéniablement liée à la nature de l'accroissement démographique de l'époque, mais également à la méthode urbanistique de concentration, dans la mise en oeuvre des ensembles de logements nouveaux.

Cette concentration est au fondement de la rentabilité qui est recherchée dans la production des logements sociaux, tant dans la constitution des réserves foncières, la réalisation de l'infrastructure (voirie et réseaux) que la construction des logements cux-mêmes. Cette conviction techniciste à courte vue va même jusqu'à concentrer les types de logement dans les immeubles, superposant ainsi les grands appartements pour rationaliser les dispositifs de construction et de distribution des fluides. L'effet mal mesuré de cette ob- 


\section{Croissance urbaine dans l'Ouest nantais}

session technicienne conduit à la formation dans les barres de quatre niveaux, de groupes d'enfants pouvant atteindre la densité insupportable de 80 individus dans chaque cage d'escalier.

\section{L'effet démographique et politique sur St-Herblain}

Un autre aspect du peuplement de la ZUP réside dans la condition sociale des habitants. Les ouvriers y dominent, $42 \%$, devançant les employés, $32 \%$; mais les professions intermédiaires n'y sont pas absentes en 1975 (17\%). Vis à vis de cette concentration populaire, dont on augure aisément le sens du vote, l'ćtablissement de la ZUP sur deux communes n'est peut-être pas sans arrière penséc. Quoiqu'il en soit le résultat de cette situation ne tardera pas à faire son effet à St-Herblain, tant au point de vue démographique que politique.

Car, si la commune enregistre, entre les deux recensements de 1968 et 1975 , le plus fort taux de croissance de son histoire (+114,4\%), passant de 18760 à 39867 habitants, clle le doit essenticllement à la création de la ZUP qui lui apporte en l'espace de sept annécs plus de 10000 habitants nouvcaux. C'est en 1975, dans l'agglomération, le plus fort pourcentage d'augmentation.

Pour leur part, les élections municipales de 1977 allaient sanctionner, en plus du poids démographique de ces nouvelles populations, lcur nouvelle sensibilité politique. Au maire à cette époque "apolitique", Michel CHAUTY ${ }^{12}$, représentatif des notables qui étaient élus dans cettc commune, jusqu'alors largement rurale, succédait un jeune maire socialiste Jean-Marc AYRAULT.

\section{L'écart entre les deux parties de la ZUP}

Les années 75-80 font apparaitue le fossé entre les deux partics de la ZUP. Il n'est pas tant lić à l'appartenance de chacune d'entre elles à Nantes ou à St-Herblain qu'a leurs caractéristiques programmatiques et sociologiques différentes.

Les densités sont ainsi plus fortes à Nantes, l'homogénéité sociale y est plus évidente (allant vers les classes les plus populaires) et l'essenticl des logements qui y sont construits $(30(0))$ sont la proprićté de l'Office Municipal d'HLM. A St-Herb'ain, même 
si le quartier reste populaire, les opérations sont plus diversifiées, mêlant l'accession à la location en même temps qu'une densité moins grande. Le "Bellevue-Nantes" fait d'ailleurs office de repoussoir pour la population herblinoise, qui le désigne comme contre-exemple et justifiera ainsi la nécessité d'aménager en espaces verts les derniers lots réservés aux promoteurs. A Nantes, la paupérisation plus accentucee de la population, favorisée par le chômage, est encore aggravée, dès 1975, par le départ des catégories sociales qui peuvent prétendre à l'accession. La chute du pourcentage des professions intermédiaires est à cet égard éloquent : il passe de $15 \%$ à $11 \%$, alors qu'à St-Herblain cette catégorie restera encore importante en 1982 (19\%).

\section{La réintégration de la partie herblinoise de la ZUP dans la réalité territo- riale de la commune}

A Saint-Herblain toute l'attention de la nouvelle municipalité de 1977 consiste à rééquilibrer le territoire de la commune de manière à faire renaître, dans les quartiers éclatés de la commune, notamment ceux qui s'étaient développés aux lisières de Nantes (le quarticr Nord et celui de la ZUP de Nantes-St-Herblain), le sentiment d'une appartenance herblinoise. C'est l'esprit qui anime la révision du POS (Plan d'Occupation du Sol) à partir de 1981. La municipalité crée à cette occasion un Atelier Municipal d'Urbanisme à la fois chargé de l'étude et de l'information des administrés. Pour susciter le débat, plusieurs scénarios seront élaborés et soumis aux herblinois au cours de différentes réunions. En 1982, le nouveau POS est définitivement adopté.

La nouvelle urbanisation envisagée s'efforce de préserver au maximum l'aspect rural de la commune. L'objectif de l'ancien POS, adopté en 1977, fixait la population communale à 100000 habitants en l'an 2000. Il est considéré comme "perspective irréaliste et inacceptable qui bétonnait la ville "13. Le nombre d'habitants à l'aube du XXIc siècle est ramené à 60000 . "Le parti d'aménagement arrêté par les élus dans le cadre du groupe de travail, est-il précisé dans le rapport de présentation du POS, a consisté à prévoir un développement d'urbanisation en quatre grands quartiers aux fonctions complémentaires, séparés les uns des autres par de vastes ensembles naturels"14. La partic herblinoise de la ZUP de Bellevue est l'un de ces quatre grands quarticrs, et le POS y confirme l'arrêt des constructions - contredisant définitivement le projet de 1961, qui tendait à intégrer la partie herblinoise de la ZUP au tissu dense de la ville de Nantes. 
Malgré la brutalité du paysage zupien et la faiblesse des différences formelles entre les parties nantaise et herblinoise de la ZUP, une série d'aménagements ont tenté, en particulier par la création de jardins et d'espaces verts (théâtre de verdure, terrain d'aventure, marché...) de distinguer l'espace herblinois au niveau du paysage urbain. Des enquêtes auprès des habitants des deux communes résidant dans la ZUP confirment l'efficacité de ces initiatives, le contraste étant encore renforcé provisoirement par le long état d'abandon de la partie nantaise ${ }^{15}$.

Après plusieurs décennies de silence, la banlicue retrouvait en quelque sorte le droit à la parole, et il semble bien que sa volonté d'cxister, non pas comme appendice de la grande ville, mais comme entité autonome, non pas digérée par la ville-centre, mais interdépendante de l'agglomération, soit pour une période suffisamment longue préservée du risque d'annexion, ou d'intégration structurelle, la subordonnant au diktat de la ville principale.

A y regarder de plus près, et ce sur une longue duréc, on remarque que le danger ou la réalité de l'intégration menace la ville de banlieue en même temps que la poussée démographique se fait plus forte et se concrétise par l'urbanisation en dehors de la villecentre. C'est la poussée démographique qui précède le siècle et produit une forte urbanisation de Chantenay qui provoque des 1905 une demande d'annexion approuvée par une loi parlementaire en 1908. De la même manière, c'est avec la poussée des années 50-60 que surgit le projet de la ZUP de Nantes-St-Herblain accompagnant l'idée d'une structure d'agglomération, niant l'existence des communes périphériques .

Somme toute, les poussées démographiques qui concernent Chantenay au XIXe siècle n'atteignent pas le taux que l'on observe à St-Herblain dans la seconde moitié du $\mathrm{XX}^{\mathrm{c}}$ siècle (+114,4 \% pour St-Herblain entre 1968 et 1975 contre $82 \%$ entre $1851 \mathrm{et}$ 1961 pour Chantenay en reprenant les plus forts taux de croissance intercensitaire). A vrai dire, bien des différences de contexte rendent ce rapprochement hasardeux, mais il nous permet de vérifier malgré tout l'intensité du crôit urbain dans la deuxième moitié de ce siècle.

Dans l'entre-deux guerres, l'annexion de Chantenay et de Doulon avait apporté à Nantes une réserve de territoire suffisante à l'expansion très modérée de la ville. A la fin 
des années 50, cette réserve était quasiment consommée, nécessitant un déploiement sur les nouvelles périphéries dont faisait partie St-Herblain .

A Chantenay, au XIXe siècle, comme à St-Herblain au $\mathrm{XX}^{\mathrm{e}}$ siècle, il est frappant de voir comment les décisions d'aménagements couvrent des projets d'intégration de la banlieue.

L'annexion n'en est pas le motif principal, il semble que les nécessités du développement rationnel de l'agglomération en soient le fondement essentiel. Toutefois, ces modalités d'extension de la ville, qu'il s'agisse de réseaux ou de quarticrs tendent à nier l'indépendance ou la cohérence territoriale de la commune limitrophe de la grande ville. Dans un second temps, ils servent d'argument à un projet d'annexion ou d'intégration structurelle. C'est sans doute ainsi que l'on peut analyser, pour Chantenay, la réalisation du Boulevard de ceinture, et, pour St-Herblain, la création de la ZUP biterritoriale de Bellevue.

Mais, en contrepartie, c'est également par des mesures d'aménagement que les responsables politiques convaincus de l'inaliénabilité de l'indépendance de leur commune, répondent également aux tentatives intégratrices. GRIVEAUD, le maire de Chantenay du début du siècle, crée une nouvelle mairie, des écoles et un lotissement au centre géométrique de sa commune de manière à en ressouder les deux parties écartelées. J.M. AYRAULT, le maire du St-Herblain d'aujourd'hui refond le plan d'occupation des sols qui redéfinit à partir de quatre quartiers existants ou à créer, un auto-développement de la commune, tout en se situant à l'intérieur de l'agglomération.

Là encore le rapprochement est hasardeux : en l'espace de trois générations la mâ̂trise de l'urbanisation s'est constituée et a construit des outils d'une relative efficacité à défaut d'une totale pertinence. L'urbanisme s'est érigé en science au début du siècle, le logement social est devenu une préoccupation communale avec la loi Bonnevay de 1912 : initiatives timides qui vont connaître au lendemain de la guerre 39-45 un développement important, mais en même temps terriblement concentré dans les mains de l'Administration de l'État, avec la loi foncière de 1953, puis celle des ZUP de 1958 et la loi d'orientation foncière de 1967 instituant les plans d'occupation des sols (POS).

Depuis le début des années 1980, ces outils juridiques de l'aménagement tendent à être réappropriés par les communes, en particulier celles qui ont créé au sein de leur 
propre administration, un service de l'urbanisme traitant l'analyse du développement communal aussi bien que sa pratique et sa prospective. A St-Herblain, l'Atelier Municipal d'Urbanisme assume cette fonction aujourd'hui, remplissant avec plus de moyens matériels et juridiques ce que faisait l'architecte-voyer de Chantenay nommé au début du siècle avec l'adoption d'un règlement sanitaire communal en 1904 et les premières mesures d'aménagement.

Pour la nouvelle commune de banlieue qu'était Chantenay à la fin du XIXe siècle, comme pris à contre pied par la brutalité relative de son développement, le court terme et le moyen terme constituent la perspective limitée de sa vision de l'avenir urbain. Les mesures d'aménagement sont prises au coup par coup, souvent dépendantes d'initiatives nantaises (on perce quelques rues nouvelles qui s'accrochent au boulevard de ceinture crée par Nantes), et la gravité de la situation du logement ne peut faire l'objet que de constats, sans qu'il y ait guère d'autre recours qu'une inflexion de l'initiative privéc en matière de réalisation des logements.

A St-Herblain, aujourd'hui, la volonté politique s'associe des moyens tels que l'Atelier Municipal d'Urbanisme et utilise des outils juridiques tels que le POS pour mâ̂triser à long terme le développement communal. La collaboration des maîtres d'ouvrage sociaux démontre par ailleurs la présence effective et solide de promoteurs immobiliers capables de prendre en compte la demande sociale et communale en matière de logements, et répondre ainsi a l'accueil de population nouvelle. Après l'épisode de la ZUP, c'est dans cet esprit qu'a ćté réalisé le quartier de Preux et qu'aujourd'hui s'édific le quartier de Tillay dans la zone dite des cinq villages. Avec le Bourg et la ZUP, ils participent des quatre grands quartiers constitutifs de la commune définis par le nouveau POS.

En même temps qu'elle marque un auto-recentrement du développement de StHerblain, cette nouvelle orientation illustre la rupture avec la soumission au diktat du toutÉtat aménageur qui, dans la région nantaise et ailleurs, a régné en maître dans les décennies 50 et 60 .

Ce diktat a produit la ZUP, réponse d'urgence à l'exode rural el au brusque cssor démographique et urbain des années 50 ou 70. Comme solution à la question du logement, la ZUP exprime la présomption des technocrates de l'État a résoudre ce problème par la production industrialiséc à grande échelle, en mécanisant le concept du confort. 
Tout un non dit de sociabilité, produit par des logiques implicites, dans de vieux quartiers apparemment anarchiques tels que Chantenay, était alors évacué dans ce type d'ensemble programmé d'en haut à partir d'une théorie réductrice et homogénéisante des besoins. Désormais, la recherche d'une plus grande proximité aux administrés dans la production de la ville réintroduit dans la forme urbaine des éléments de diversité, de spécificité et de localité que ne contenaient aucunement les ZUP.

Enfin, malgré les tensions persistantes entre la banlieue et la ville centre ${ }^{16}$ résultant des divergences et rivalités politiques et politiciennes et de la concurrence souvent acharnée dans les tentatives de captation des grands projets d'aménagement, la conscience d'une solidarité d'agglomération existe aujourd'hui, coexistant avec la volonté de préservation et d'autodéveloppement de la banlieue. A St-Herblain, cette attitude domine alors que dans l'agglomération nantaise du début du XXe siècle, la gucrre entre la banlicue de Chantenay et la grande ville ćtait à coutcaux tirés.

\section{a NOTES}

1. Voir L'indépendance confisquée d'une ville ouvrière, Chantenay, Nantes, F́ditions ACI.. 1982

2. Voir "Bellevue, une ZUP", Nantes, ouvrage à parâtıe aux Éditions $\Lambda \mathrm{CL}$.

3. Cf Marcel RONCA YOLO, Histoire de la France urbaine, Paris, Éditions du Seuil, t. $4:$ La ville de l'âge industriel, 1983, p. 29.

4. C. MELLINET, "La Commune et la Milice de Nantes", Nantes, Imp. MLLLINET, 1840.

5. Voir D. PINSON : "Le rapport industric/habitat à Chantenay", in Villes en Parallèles, $n^{\circ} 10$, Université de Paris X, Nanterre, Juin 1986, pp. 172-185.

6. Nantes Social, Nantes, Centre de Documentation des Musćes du Château, 1922-1925.

7. "Nantes Saint-Nazaire", La Documentation Française , $\mathrm{n}^{\circ}$ 3362, Paris, 1967.

8. Notice explicative de l'Enquête d'utilité publique en vue de la création de la ZUP de Nantes-StHerblain (ADLA 147 W 278)

9. ADLA 147 W 78, AMN et AMSH.

10. Voir E. PRETECEILLE, La production des grands ensembles, Paris, La Haye, Éditions MOUTON, 1973.

11. En particulier celle qui impose à l'Office HLM de la Ville de Nantes de ne construire que sur le sol de son autorité de tutelle. 
12. En 1985, Michel CHAUTY, désormais affilié au R.P.R., a été élu maire de Nantes, battant l'ancien maire socialiste Alain CHENARD.

13. Rapport de présentation du P.O.S. de St-Herblain (1982).

14. Idem.

15. Une réhabilitation d'envergure et particulièrement visible a toutefois été engagéc en 1987.

16. Et même entre banlieues, cette concurrence se manifestant aujourd'hui autour de la création, à la périphéric de l'agglomération, de vastes zones commerciales. 


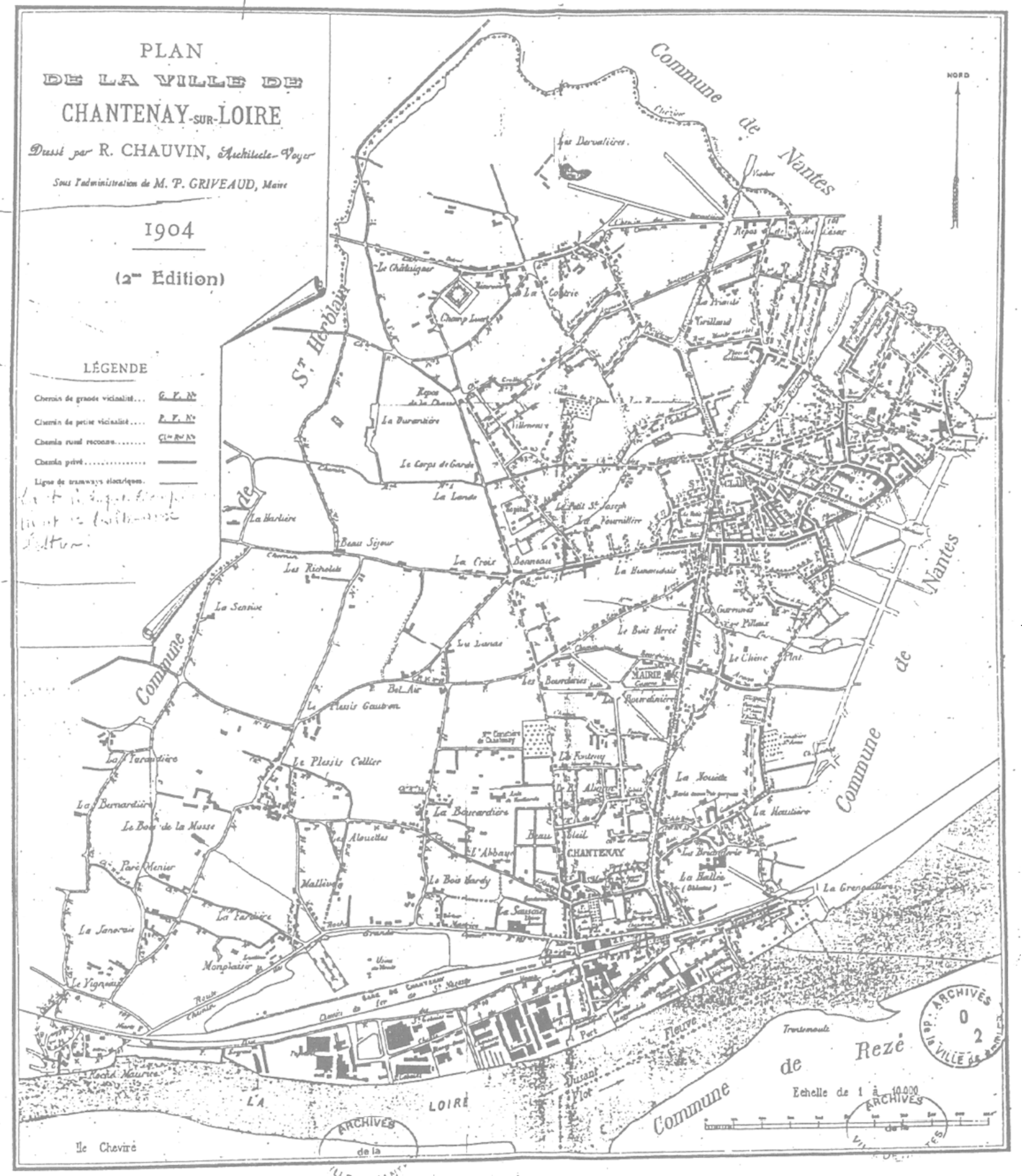

Plan de la ville de Chantenay sur Loire (1904) faisant apparaître les masses bâties 


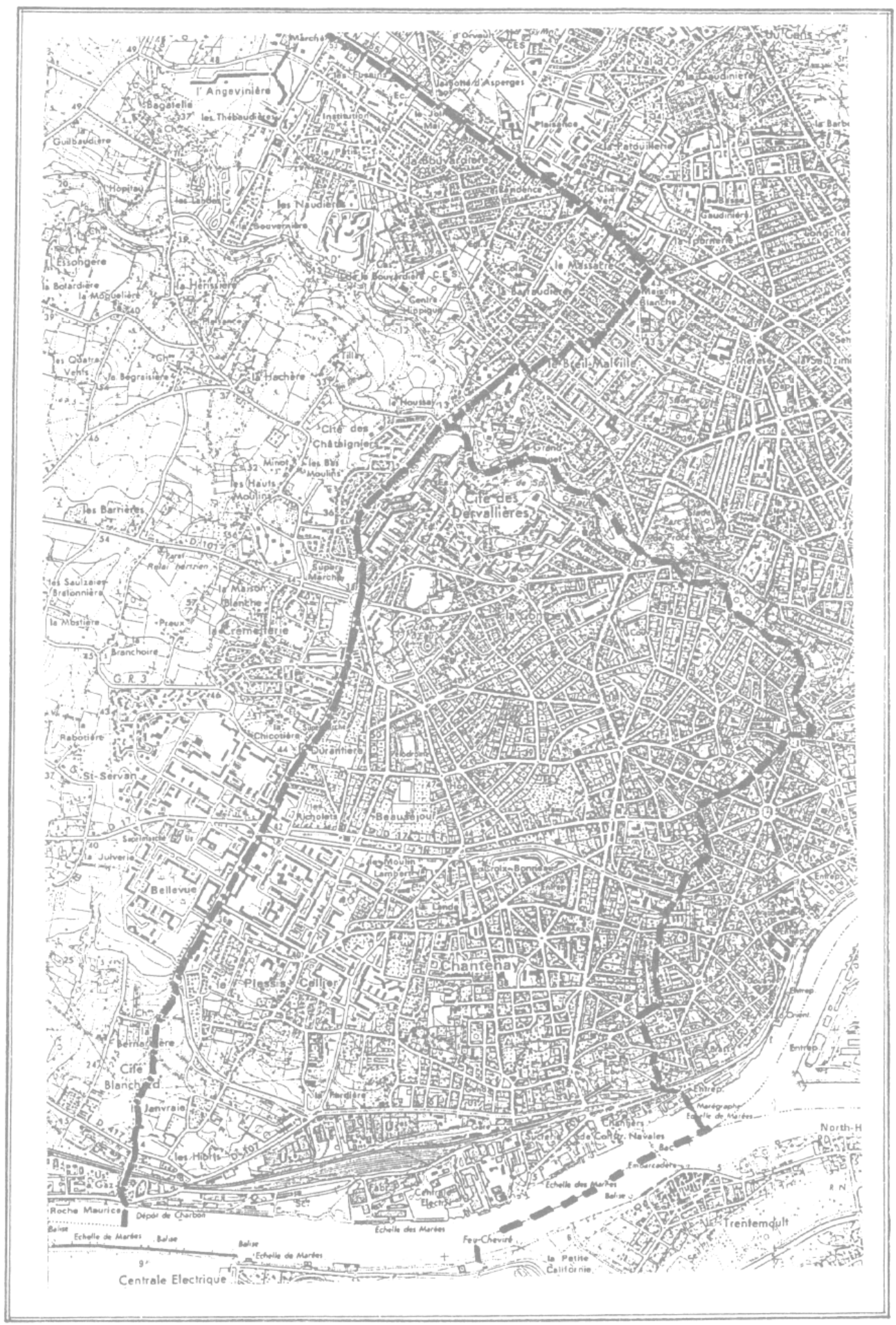

Extrait de la carte topographique de l'IGN au 1/25000e (1974) avec mise en évidence des limites de l'ancienne commune de Chantenay, de Nantes et de Saint-Herblain 


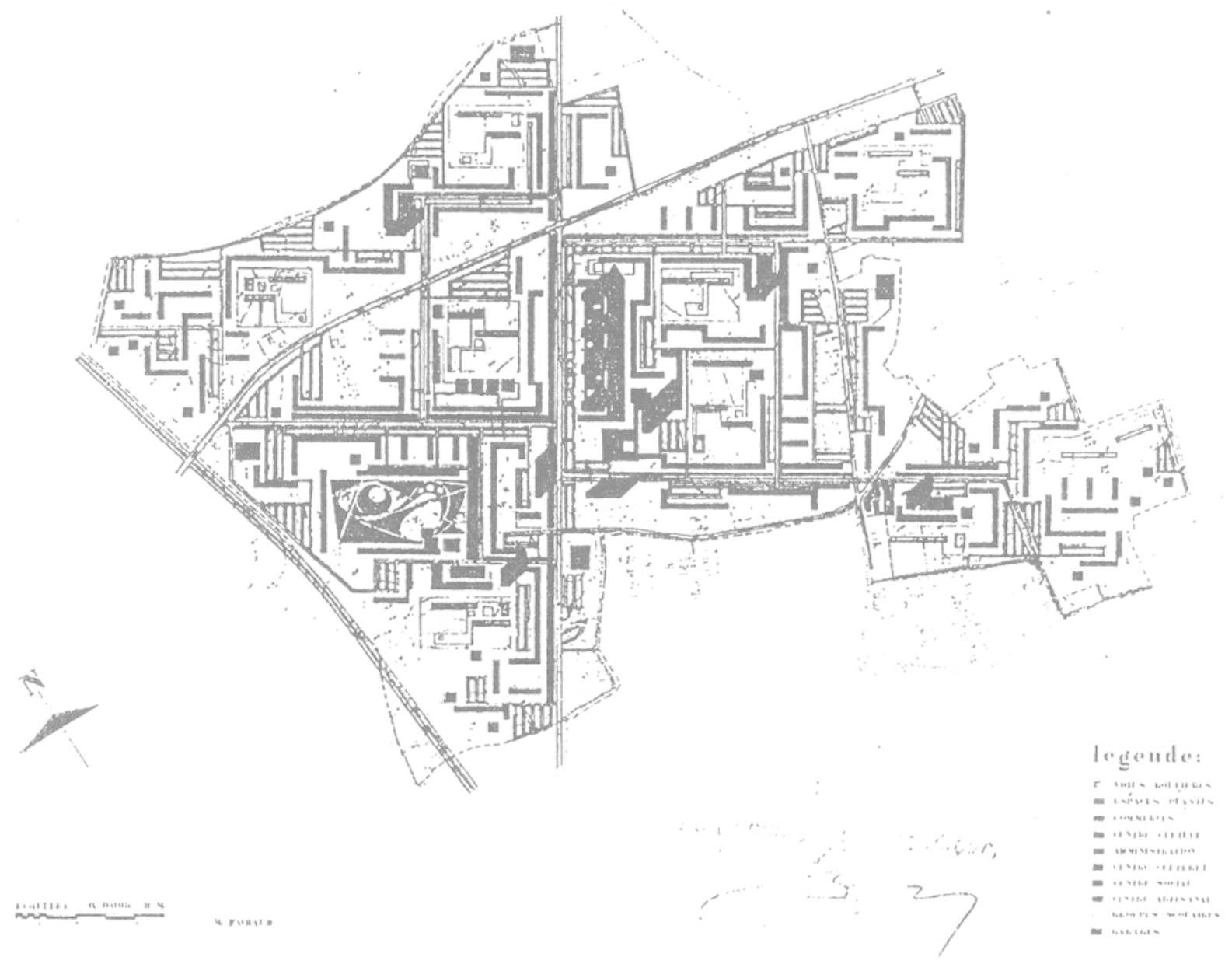

Plan de la ZUP de Nantes Saint-Herblain élaboré par l'architecte Marcel FAVRAUD pour la Société d'Équipement de Loire-Atlantique (SÉLA), 1962 


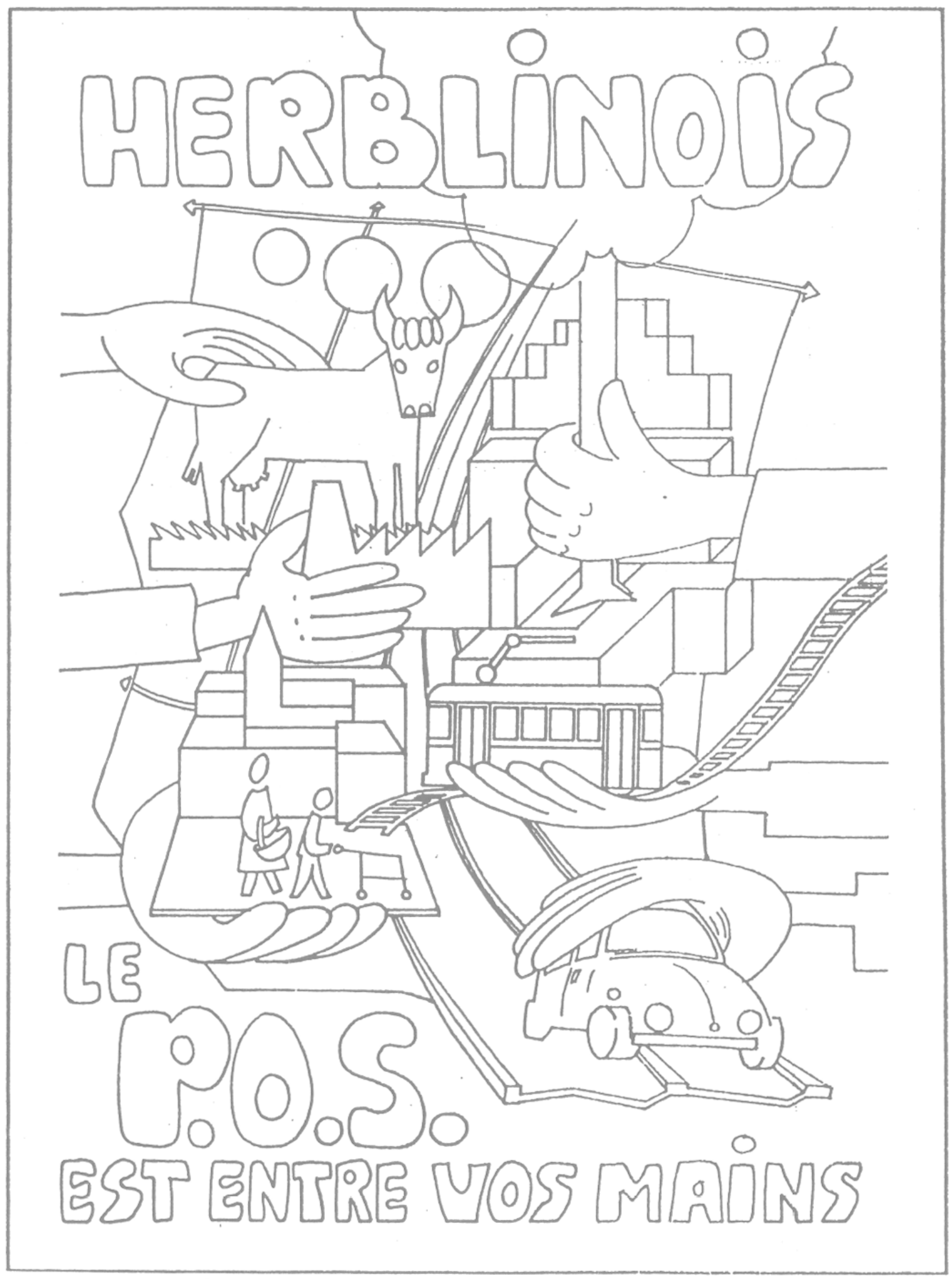

Source : "Vivre à Saint-Herblain", bulletin municipal, 1981 


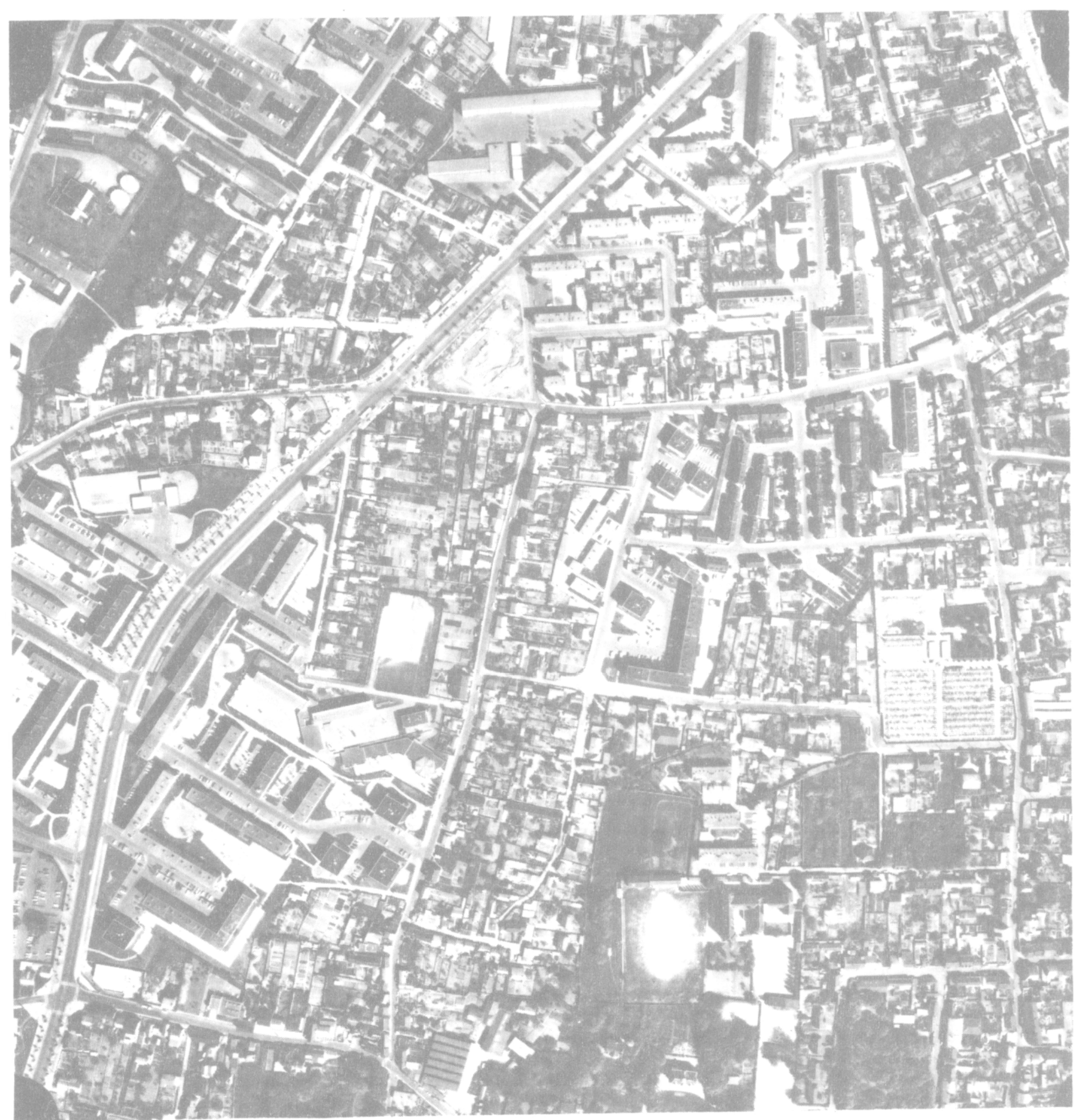

